\title{
Registro de Neuroseñales con una Interfaz Cerebro-Computador para Estimar el Nivel Estrés en un Estudiante durante una Clase
}

\section{Neurosignal Record with a Brain- Computer Interface to Estimate the Level of Stress in a Student During a Class}

DOI: http://doi.org/10.17981/ingecuc.13.2.2017.10

Artículo de investigación científica. Fecha de recepción: 16 septiembre de 2017 Fecha de aceptación: 9 de noviembre de 2017

\author{
Luz Angela Moreno Cueva \\ Universidad de Pamplona. Pamplona (Colombia) \\ luz.moreno@unipamplona.edu.co \\ César Augusto Peña Cortés \\ Universidad de Pamplona. Pamplona (Colombia) \\ cesarapc@unipamplona.edu.co \\ Marisol Maestre Delgado \\ Universidad de Pamplona. Pamplona (Colombia) \\ marisol.maestre@unipamplona.edu.co

\section{Surgei Bolivia Caicedo Villamizar \\ Universidad de Pamplona. Pamplona (Colombia) subocavi@unipamplona.edu.co} \\ Aldo Pardo García \\ Universidad de Pamplona. Pamplona (Colombia) \\ apardo13@unipamplona.edu.co
}

Para citar este artículo:

L.A. Moreno Cueva, C.A. Peña Cortés, M. Maestre Delgado, S.G. Caicedo Villamizar, A. Pardo García, "Registro de neuroseñales con una interfaz cerebro-computador para estimar el nivel estrés en un estudiante durante una clase," INGE CUC, vol. 13, no. 2, pp. 95-101, 2017. DOI: http://doi.org/10.17981/ingecuc.13.2.2017.10

\section{Resumen}

Introducción- El presente trabajo muestra un estudio de carácter individual que captura, registra y analiza el nivel de estrés de un estudiante universitario durante una clase que involucra una evaluación. La información de estrés se estimó por medio de una interfaz cerebro-computador comercial y de bajo costo. Esto permite solucionar el problema de obtener fácilmente medidas cuantitativas y no solo cualitativas.

Objetivo- Analizar el comportamiento de las neuroseñales para estimar el nivel de estrés en un estudiante ante algunos eventos verbales y no verbales generados por un docente.

Metodología-Se desarrolló un diseño experimental de carácter individual tomando como perturbaciones al nivel de estrés eventos tales como preguntas, limitaciones de tiempo y gestos.

Resultados-Se logró poner en evidencia algunos eventos que causan estrés en los estudiantes producidos por el lenguaje verbal y no verbal del docente al impartir la clase.

Conclusiones-Se recomienda a los docentes moderar su lenguaje corporal durante las evaluaciones evitando acciones que emulen afanes o presiones en tiempos innecesarios.

Palabras clave- Neuroseñales, estrés, clase, educación, emociones, ICC

\section{Abstract}

Introduction- This work shows an individual study of the capture, recording, and analysis of the level of stress of a university student during a class that involves an evaluation. The stress information was estimated using a commercial and low-cost computer-brain interface. This allows solving the problem of easily obtaining quantitative and not only qualitative measures.

Objective-The aim of this article is to analyze the behavior of neural signals to estimate the level of stress in a student to some verbal and nonverbal events generated by a teacher.

Methodology- An experimental design of individual character was developed taking as disturbances the level of stress, events such as questions, time limits, and gestures.

Results-Some events that caused stress in students produced by the verbal and non-verbal language of the teacher when teaching the class were evidenced.

Conclusions- Teachers are encouraged to moderate their body language during assessments by avoiding actions that emulate anxieties or pressures in unnecessary times.

Keywords- Neurosignals, stress, class, education, emotions, BCI 


\section{INTRODUCCIÓN}

Desde hace varios años, el estrés se ha considerado una enfermedad que se incrementa con el paso de los días, comenzando por aquel que es generado por las grandes cargas laborales [1]-[3]we model for the first time a number of qualitative variables as features which represent crisis (especially stress and dissatisfaction but also migration, reprioritizing and behavioral changes, las responsabilidades familiares [4], las deudas, la edad [5], hasta el estrés de los niños dentro de las responsabilidades que derivan de sus escuelas [6] debido a la presión que en ocasiones ejercen los maestros e, incluso, los padres que buscan la excelencia de sus hijos.

La RAE define el estrés como la "tensión provocada por situaciones agobiantes que originan reacciones psicosomáticas o trastornos psicológicos a veces graves" [7], es por ello, que se hace necesario determinar de forma precisa aquellas acciones que realiza el maestro dentro de una clase para no generar esas situaciones que pueden producir problemas en cada uno de los aprendices.

Las personas, sin importar la edad, pueden sufrir tres tipos de estrés: el agudo, el agudo episódico y el crónico; la diferencia en ellos radica en el tiempo en el que se dan, siendo el primero a corto plazo y el último a largo plazo [8].

Para evitar inconvenientes a tan temprana edad se han desarrollado diferentes técnicas que permiten medir el estrés presentado en los estudiantes, como el Inventario de Estrés Académico [9], siendo implementadas en diversas investigaciones en jóvenes de secundaria y en programas de pregrado.

Con el paso de los años se ha visto necesario conocer de forma biológica y cuantitativa los niveles de estrés y otras emociones que, de cierta forma, se involucran y contribuyen al incremento de ella, para así tener mayor veracidad en cada uno de los estudios realizados.

Las imágenes por resonancia magnética funcional, fMRI, y la lectura electroencefalográfica han tomado gran importancia en la evaluación de la actividad cerebral, pues allí, gracias a las distintas emociones que percibe la persona de su entorno, se concibe el estrés.

En el caso de entornos educativos, esas emociones pueden llegar ser acusadas o afectadas según la forma como interactúan el estudiante y el maestro, las estrategias pedagógicas [10], los problemas familiares que se pueden presentar por las calificaciones, la presión de respuestas óptimas esperadas [11], los precisos momentos en donde se limpia un tablero cuando el sujeto en investigación no ha terminado de transcribir en su lugar de apunte, los estereotipos que generan los compañeros, el leguaje tanto verbal como no verbal (lenguaje corporal) por parte del docente, entre otras.
En este trabajo se pretende medir o cuantificar, por medio de un estudio individual, el estrés que puede sufrir un estudiante en una clase cuando el docente realiza una serie de eventos verbales y no verbales. Estás mediciones se realizan por medio de una interfaz cerebro-computador de bajo costo que se basa en técnicas de electroencefalografía. Este estudio puede servir como ejemplo o punto de partida para analizar acciones o generar eventos en entornos pedagógicos y corroborar grosso modo su impacto emotivo sobre los estudiantes.

\section{Metodología Para la CAPtura de NeUroseñales}

Las interfaces BCI (Brain Computer Interface), en sus orígenes, eran bastante costosas, pero con el paso del tiempo se han desarrollado diferentes instrumentos de éste tipo, tales como: MindFlex de Mattel [12], MindSet de NeuroSky [13], Muse de InteraXon [14] y el Emotiv EPOC e Insight, que disminuyen los costos de forma considerable, permitiendo el incremento en su uso en investigaciones en otros ámbitos de las ciencias, como en el marketing con el análisis de pautas publicitarias [15], en pedagogía para detectar problemas para mejorar los estilos de aprendizaje de estudiantes de ingeniería [16], en robótica con el control mental de los robots [17], entre otros. Éstos han tenido gran acogida porque con ellos se obtienen resultados de mayor precisión pues se registran las actividades cerebrales inconscientes de la persona, donde ya se conoce que surge la gran mayoría de la toma de decisiones y de la determinación de las acciones.

Para efectos del presente trabajo se tiene en cuenta el dispositivo Emotiv Insight (Fig. 1) dado que es cómodo para el individuo que se está evaluando [18]. Posee cinco sensores de polímero hidrófilo, lo que significa que no debe ser humedecido para la conducción de las ondas. Dos de los sensores son de referencia, logrando obtener una mejor ubicación que permite la medición de seis tipos de emociones, los cuales son: relajación, estrés, concentración, compromiso, entusiasmo e interés, tanto en lapsos cortos como largos [19].

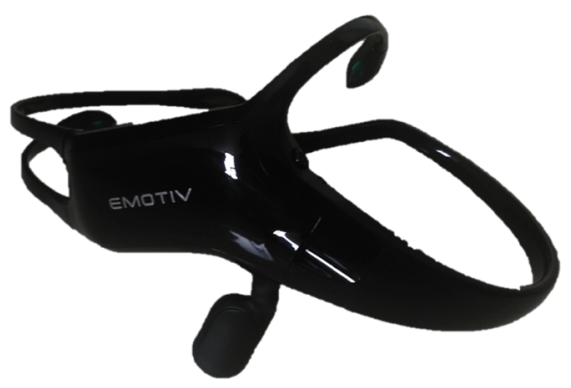

Fig. 1. Emotiv Insight. Fuente: Autores 
El dispositivo permite un uso continuo aproximado de 4 horas, sin embargo, no es recomendable su uso por extensos periodos dado que la presión que ejercen los sensores sobre la cabeza puede resultar molesta para algunos usuarios. Otra situación que se debe tener en cuenta es que el dispositivo no puede estar alejado del dispositivo bluetooth, de lo contrario, se pierde la conectividad.

\section{A. Descripción de la Interfaz de Captura de Neuroseñales}

El Emotiv Insight captura las señales eléctricas que emite el cerebro ante una actividad o pensamiento que desarrolla el sujeto evaluado [20].

Dentro de sus funciones se encuentra el reconocimiento de gestos faciales, interfaz que identifica gestos como la sonrisa, los guiños, los movimientos de ojos, el fruncimiento del ceño, apretar dientes [21], entre otros [22]. En la Fig. 2 se puede apreciar una ilustración de la interfaz mencionada.

Otra parte de la interfaz es el reconocimiento cognitivo. Éste permite hacer el reconocimiento de comandos mentales luego de un entrenamiento. Una de las aplicaciones más usadas de esta interfaz es para operar dispositivos (carros, drones, robots, sillas de rueda, etc.) a través de los comandos mentales adelante, atrás, izquierda, derecha, subir y bajar (Fig. 3).

En la Fig. 4 se puede apreciar la interfaz en el modo de reconocimiento de estados emotivos. Éste modo permite detectar los estados en una escala normalizada (0 a 1).

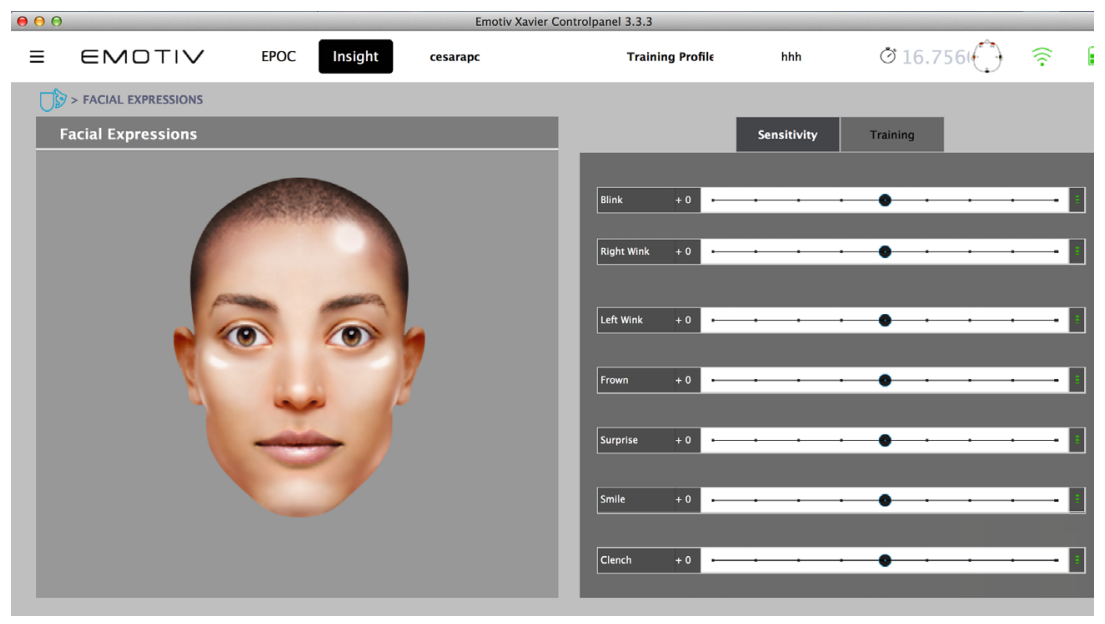

Fig. 2. Reconocimiento de gestos faciales capturados con el panel de control de Emotiv (Xavier). Fuente: Autores.

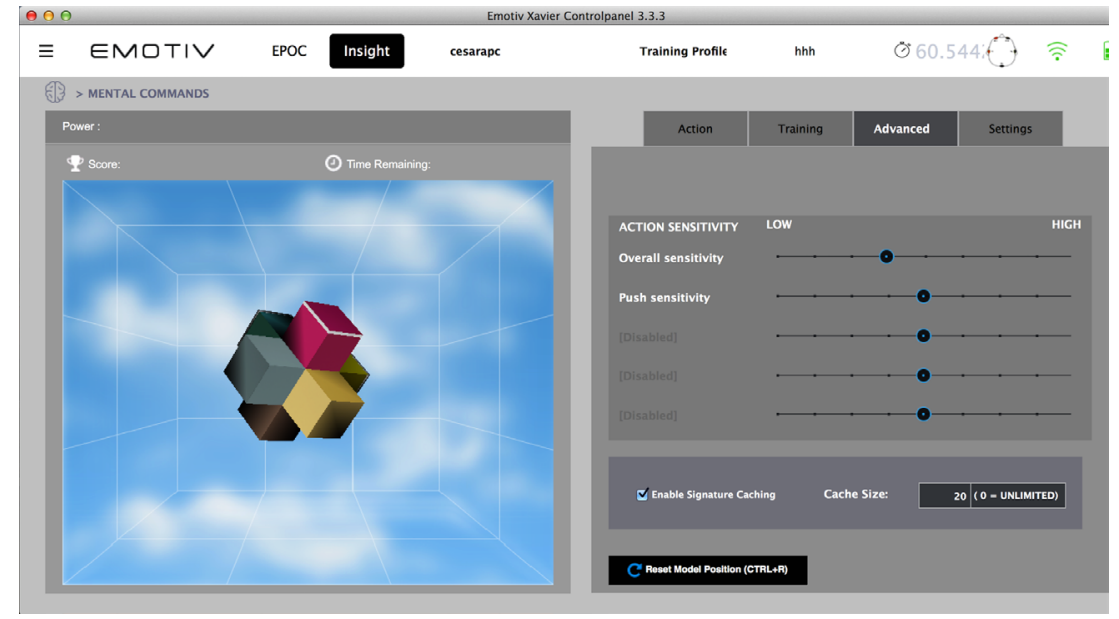

Fig. 3. Reconocimiento de comandos mentales o gestos cognitivos capturados con el panel de control de Emotiv (Xavier). Fuente: Autores. 


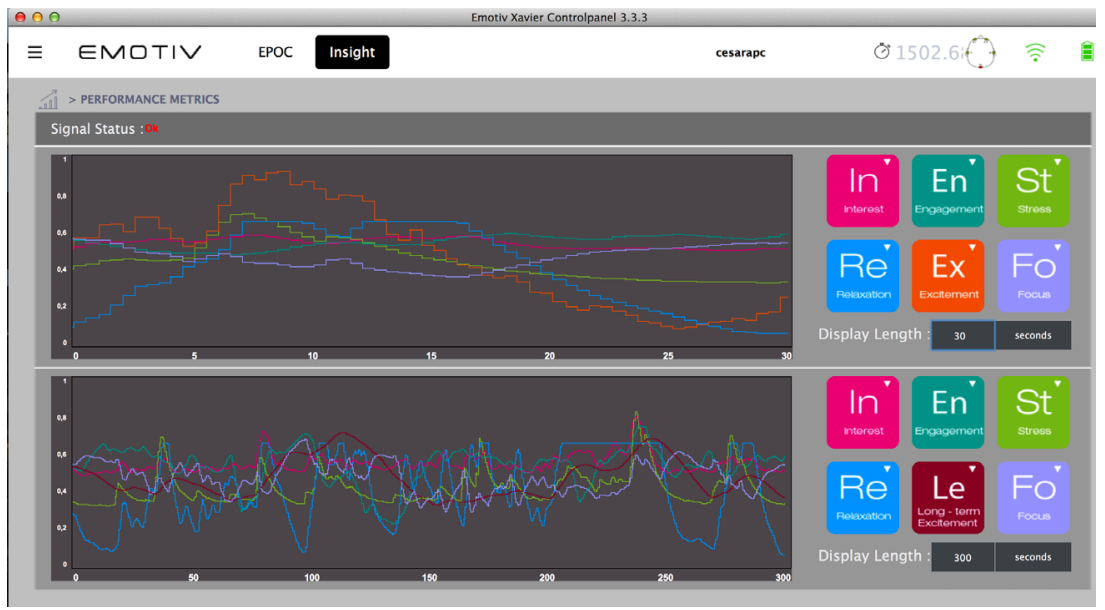

Fig. 4. Registro emotivo capturado con el panel de control de Emotiv (Xavier). Fuente: Autores.

Los estados emotivos se pueden medir a corto y largo plazo y en diferentes escalas de tiempo, por lo cual la interfaz gráfica se encuentra dividida en dos. Los estados emotivos que se pueden estimar son: interés, compromiso, estrés, relajación, excitación o entusiasmo y concentración, como ya se había mencionado.

\section{B. Planeación del Experimento}

Con el fin de buscar evidencias del estrés en un aprendiz, se procedió al registro de las neuroseñales de un estudiante mientras se desarrolla una clase con el tema de diversidad. Con el fin de tener más eventos del estrés se planeó dentro de la clase una evaluación. Cabe mencionar que esta evaluación no tenía un significado en notas, dado que la clase no se relacionaba con un curso directo, sino que era un joven de un semillero de investigación. Se escogió de esta forma porque se quería conocer las emociones del estudiante frente a estos retos, mas no se quería presionar con una nota. De hecho, la evaluación era un ejercicio didáctico sobre encontrar palabras, denominado popularmente como sopa de letras.

Una vez el dispositivo se instale en el usuario, se deberá calibrar. Para lograrlo, se debe verificar el correcto funcionamiento de los sensores, así como también requiere de una calibración con los ojos abiertos y otra con los ojos cerrados durante determinado tiempo. Posteriormente, se observará el funcionamiento del dispositivo en un tiempo en donde no se realizará evaluación de datos con respecto al experimento como tal, pues inicialmente la persona que se encuentra haciendo uso del Emotiv Insight estará ansioso e inquieto por estar utilizando el dispositivo. En la Fig. 5, se puede apreciar el estado emotivo que tiene el estudiante cuando comienza la clase.

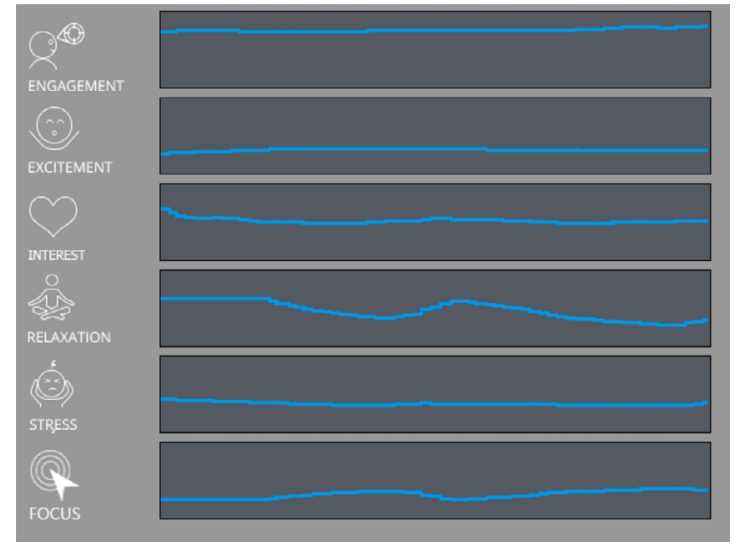

Fig. 5. Estado emotivo inicial capturado con el panel de control de Emotiv (Xavier) del estudiante al iniciar la clase. Fuente: Autores.

\section{III.RESUltados}

En ésta sección se muestran los resultados obtenidos de la planeación del experimento. Luego de pasar la etapa de habituación del estudiante al dispositivo, se pudo corroborar que éste iniciaba la clase con un nivel de estrés bajo. Para poner en evidencia si existían cambios en los estados emotivos del sujeto, la docente realizó preguntas. En la Fig. 6 se puede apreciar en la señal del estrés cómo fluctuaron los niveles cuando el docente le realizó preguntas (ver resaltos). Debe tenerse en cuenta que el incremento del estrés usualmente surge cuando los estudiantes no cuentan con suficiente confianza, no conocen la temática con propiedad, sienten pena al hablar en público, es presionado para entregar trabajos, es evaluado delante de los compañeros, entre muchas otras circustancias. 


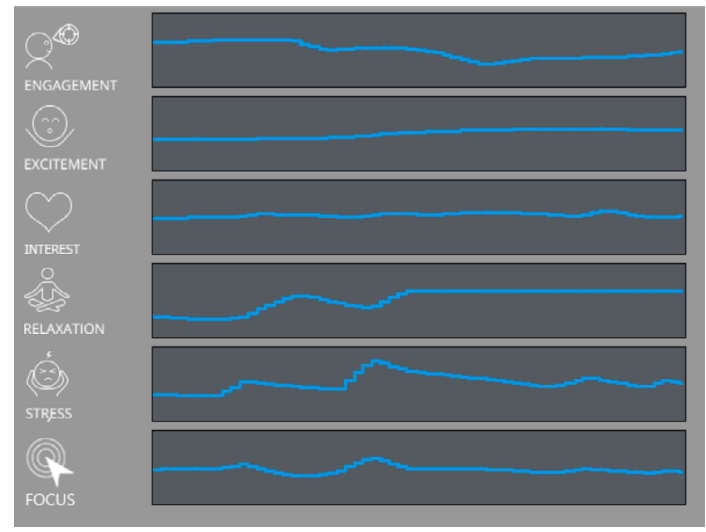

Fig. 6. Estado emotivo del estudiante capturado con el panel de control de Emotiv (Xavier) al realizarle una pregunta. Fuente: Autores.

En la Fig. 7 se puede apreciar que los niveles emotivos del estudiante se estabilizan y son relativamente uniformes cuando el docente se hace cargo de la clase sin solicitar intervenciones por parte del estudiante; se puede observar cómo la señal de estrés vuelve a niveles relativamente bajos.

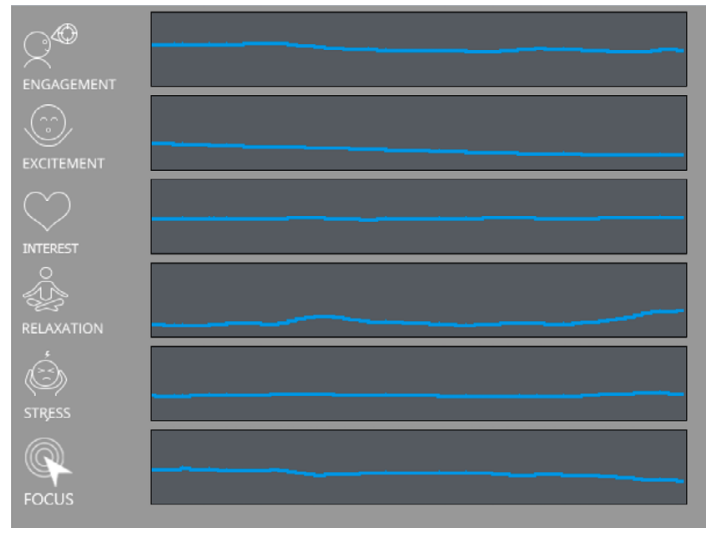

Fig. 7. Estado emotivo del estudiante capturado con el panel de control de Emotiv (Xavier) mientras escucha a la docente. Fuente: Autores.

Con estas pequeñas pruebas se pone en evidencia que el sistema de captura de neuroseñales registra los eventos producidos durante la clase a través de las emociones del estudiante examinado.

A continuación, se muestran los resultados correspondientes al lapsus final de la clase, correspondiente a la evaluación del estudiante por medio de un ejercicio didáctico donde él tiene que encontrar y señalar las palabras solicitadas (ejercicio sopa de letras).

De acuerdo con el cronograma del experimento, la docente le pregunta al estudiante cuántas respuestas lleva de la prueba. En la Fig. 8 se puede observar que el estudiante eleva momentáneamente sus niveles de estrés pese a que le queda bastante tiempo para culminar con la prueba.

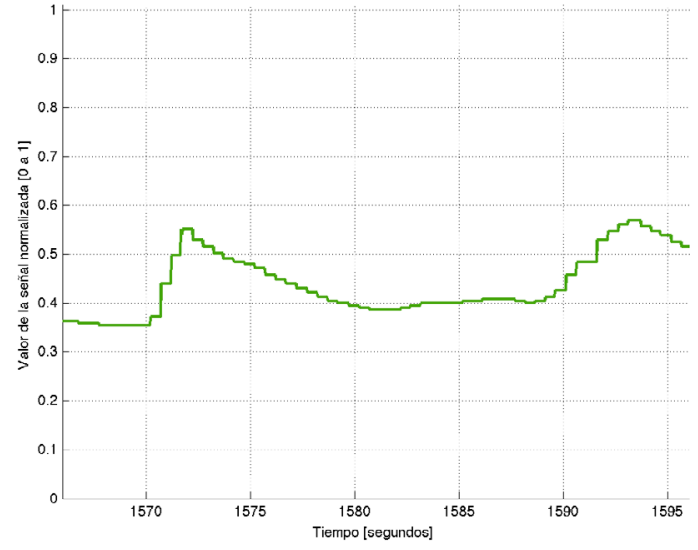

Fig. 8. Nivel de estrés cuando la docente le pregunta al estudiante cuántas respuestas lleva de la prueba.

Fuente: Autores.

Debe tenerse en cuenta que el lenguaje no verbal juega un papel muy importante en las clases que imparte un docente. Por ejemplo, en la Fig. 9 se observa el registro del nivel de estrés ante dos eventos: el primero, el cual ocurre a los 1907 segundos, donde el docente vuelve a preguntarle al estudiante sobre la prueba y éste eleva de forma tenue el estrés; el segundo evento (1938s) corresponde a que la docente se coloca de pie, apaga y cierra su computador personal. Se puede apreciar cómo ese gesto eleva significativamente el estrés en el estudiante (nuevamente se aclara que el estudiante cuenta aún con tiempo para culminar la prueba).

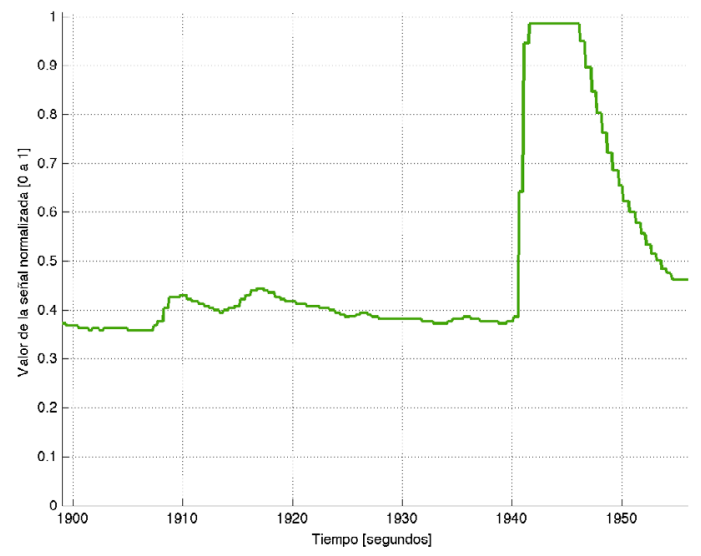

Fig. 9. Profesor pregunta y apaga el computador. Fuente: Autores.

Minutos después, tiempo suficiente para que el aprendiz haya completado la prueba (el tiempo dado para finalizar la prueba fue más del requerido para hacerlo), él mismo da un suspiro y hace el comentario en voz alta de que no encuentra más respuestas. Se pueden observar, en la Fig. 10 de los diferentes estados emotivos ante esta reacción, cómo cambian las señales de estrés, relajación, interés y concentración. 


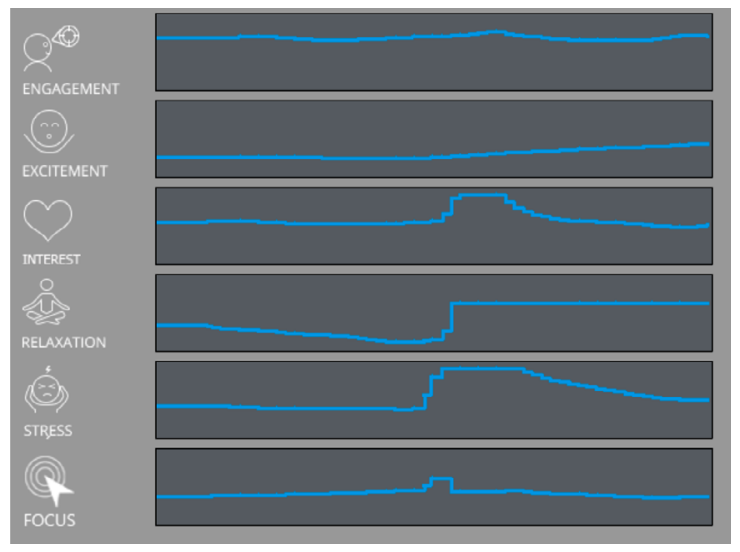

Fig. 10. Estudiante suspira y menciona no encontrar más respuestas.

Fuente: Autores.

En la Fig. 11 se ilustran los resultados correspondientes a la finalización y entrega de la prueba. En primer lugar, el individuo en evaluación vuelve a suspirar $(2252 \mathrm{~s})$, posteriormente, la docente le comunica que terminó el tiempo (2285s) y que la prueba le queda para resolverla en casa.

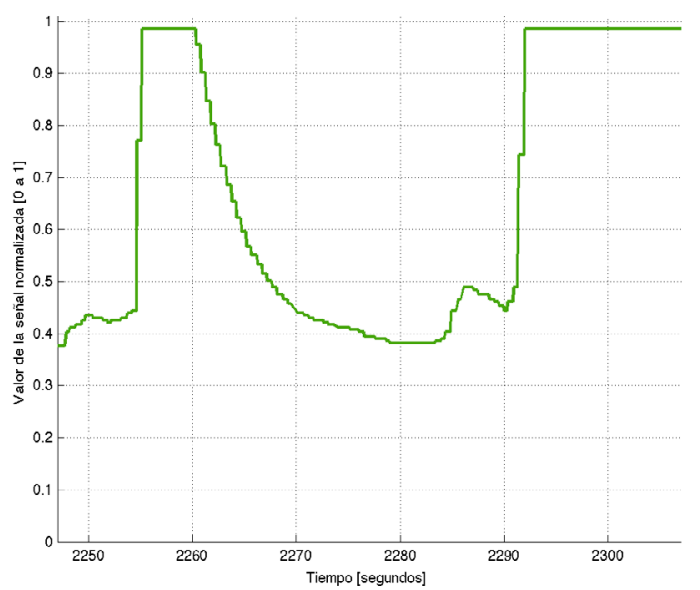

Fig. 11. Estudiante vuelve a suspirar y le comunican que culminó el tiempo de la prueba.

Fuente: Autores.

Tabla 1. Nivel de estrés frente a los estímulos generados.

\begin{tabular}{|l|l|l|}
\hline \multicolumn{1}{|c|}{ Estimulo o Evento } & \multicolumn{1}{c|}{ Valor } & \multicolumn{1}{c|}{ Observación } \\
\hline Escuchando la clase & 0,350 & Nivel bajo \\
\hline $\begin{array}{l}\text { Primera pregunta en } \\
\text { medio de la prueba }\end{array}$ & 0,652 & Nivel medio \\
\hline $\begin{array}{l}\text { Segunda pregunta en } \\
\text { medio de la prueba }\end{array}$ & 0,429 & Nivel bajo \\
\hline $\begin{array}{l}\text { Docente se para y apaga } \\
\text { el computador (antes de } \\
\text { terminar el tiempo) }\end{array}$ & 0,984 & Nivel muy alto \\
\hline Termina el tiempo & 0,987 & Nivel muy alto \\
\hline
\end{tabular}

Fuente: Autores.
En la tabla 1, se presentan, de forma resumida, los niveles de estrés del estudiante ante los diferentes estímulos generados. Puede ponerse en evidencia el correcto funcionamiento del sistema, además de la importancia del lenguaje no verbal del docente.

\section{Conclusiones}

Con este experimento se pudo corroborar que el dispositivo de captura de neuroseñales Emotiv Insight, pese a que es un elemento de bajo costo, logra reflejar algunos cambios en los estados emotivos del estudiante.

A pesar de que el ejercicio que planteaba el experimento no involucraba una nota en el estudiante ni le representaba ningún tipo de calificación que afectara su record académico, se logró demostrar que algunos eventos pueden causar o generarle algún tipo de estrés; uno de los principales detectados fue el gesto por parte de la docente de cerrar el computador antes de finalizar la prueba. Esto puede generar un aprendizaje, que es el de no distraer a los alumnos mientras realizan sus pruebas. En muchas ocasiones, los docentes se afanan, borran el tablero, alistan sus bolsos, guardan sus dispositivos electrónicos para la enseñanza como el video beam y se intuye que todos estos eventos pueden afectar el nivel de estrés del estudiante.

Por otra parte, se pudo poner en evidencia el aumento de los niveles de estrés en las intervenciones verbales del estudiante. Se propone, en un trabajo futuro, hacer un estudio de los niveles de estrés con respecto a preguntas e intervenciones en temas dominados plena, mediana y bajamente por el alumno.

En conclusión, se puede interpretar que el uso de las nuevas tecnologías en las neurociencias y la disminución de los costos de los dispositivos tecnológicos asociados permiten hacer una evaluación, por lo menos de forma aproximada, de los estados emotivos de los estudiantes frente a las estrategias didácticas implementadas por los docentes.

Dados los buenos resultados individuales, se propone extender el estudio, tomando una muestra significativa de estudiantes que permitan obtener un gran número de datos experimentales con varias repeticiones permitiendo estimar el comportamiento emotivo sobre una población en particular.

\section{Financiamiento}

Artículo de investigación científica derivado del proyecto de investigación titulado "Análisis de neuroseñales en la aplicación de estrategias pedagógicas y/o didácticas", financiado por la Universidad de Pamplona. Fecha de inicio: enero de 2017. Fecha de finalización: diciembre 2017. 


\section{REFERENCIAS}

[1] G. Halkos y D. Bousinakis, "The effect of stress and dissatisfaction on employees during crisis," Econ. Anal. Po licy, vol. 55, pp. 25-34, 2017. http://dx.doi.org/10.1016/j. eap.2017.04.002

[2] R. Repetti y S. Wang, "Effects of job stress on family relationships," Curr. Opin. Psychol., vol. 13, pp. 15-18, 20. http://linkinghub.elsevier.com/retrieve/pii/ S2352250X16300240

[3] A. Marchand, J. Bilodeau, A. Demers, N. Beauregard, P. Durand y V. Y. Haines, "Gendered depression: Vulnerability or exposure to work and family stressors?," Soc. Sci. Med., vol. 166, pp. 160-168, 2016. DOI: 10.1016/j. socscimed.2016.08.021

[4] A. S. Masarik y R. D. Conger, "Stress and child development: A review of the Family Stress Model," Curr. Opin. Psychol., vol. 13, pp. 85-90, 2017. http://dx.doi. org/10.1016/j.copsyc.2016.05.008

[5] A. A. Stone, S. Schneider y J. E. Broderick, "Psychological stress declines rapidly from age 50 in the United States: Yet another well-being paradox," J. Psychosom. Res. vol. 103, 2017. http://linkinghub.elsevier.com/retrieve/ pii/S0022399917309716

[6] C. Salavera, P. Usán, S. Pérez, A. Chato y R. Vera, "Differences in Happiness and Coping with Stress in Secondary Education Students," Procedia - Soc. Behav. Sci., vol. 237, pp. 1310-1315, 2017. http://linkinghub.elsevier. com/retrieve/pii/S187704281730215X

[7] RAE, "Diccionario de la Real Academía la Lengua Es pañola," 2017. [En línea]. Disponible en: http://dle.rae. es/?w=estres.

[8] Association American Psychological, "Los distintos tipos de Estrés," 2017. [En línea]. Disponible en: http://www. apa.org/centrodeapoyo/tipos.aspx

[9] C. Fernández Jiménez y M. T. Polo Sánchez, "Afrontamiento, estrés y bienestar psicológico en estudiantes de educación social de nuevo ingreso," EduPsykhé. Rev. Psicol. y Educ., vol. 10, no. 2, pp. 177-192, 2011. https:// dialnet.unirioja.es/servlet/articulo?codigo $=3896794$

[10] T. Velásquez Pérez, E. E. Espinel Blanco y G. Guerrero Gómez, "Estratégias Pedagógicas en el aula de clase," Rev. Colomb. Tecnol. Av., vol. 2, no. 28, pp. 124-131, 2016. http://www.unipam plona.edu.co/unipamplona/ portalIG/home_40/recursos/revistas/20052017/revista_28.jsp

[11] I. S. Flórez, L. E. Mendoza y V. Pérez Ginna, "Medición automática del nivel de aprendizaje en niños de 4 a 6 años de edad aplicando técnicas de procesamiento de señales," Rev. Colomb. Tecnol. Av., vol. 2, no. 28, pp. 93-100, 2016. http://www.unipamplona.edu.co/unipamplona/portalIG/ home_40/recursos/revistas/20052017/revista_28.jsp

[12] J. Katona, I. Farkas, P. Ujbanyi, A. Dukan y A. Kovari, "Evaluation of the NeuroSky MindFlex EEG headset brain waves data," in IEEE 12th International Symposium on Applied Machine Intelligence and Informatics (SAMI), pp. 91-94, 2014. http://ieeexplore.ieee.org/document/6822382/
[13] A. Fernández y M. Eid, "Read Go Go!: Towards real-time notification on readers' state of attention," in XXIV International Symposium on Information, Communication and Automation Technologies (ICAT), pp. 1-6, 2013. http://ieeexplore.ieee.org/document/6684047/

[14] W. A. Dijk, W. van der Velde, W. J. M. Kolkman, H. J. G. M. Crijns y K. I. Lie, "Integration of the Marquette ECG management system into the Department Information System using the European SCP-ECG Standard," Computers in Cardiology, 1995. [En línea]. Disponible en: http://ieeexplore.ieee.org/document/482669/

[15] L. Moreno, C. Peña y H. González, "Integración de un sistema de neuroseñales para detectar expresiones en el análisis de material multimedia," Rev. Fac. Ing., vol. 24, no. 38, pp. 29-40, 2014. http://revistas.uptc.edu.co/ index.php/ingenieria/article/view/3156/4343

[16] F. M. Córdova, H. Díaz M., F. Cifuentes L. Cañete y F. Palominos, "Identifying problem solving strategies for learning styles in engineering students subjected to intelligence test and EEG monitoring," Procedia Comput. Sci., vol. 55, pp. 18-27, ITQM, 2015. Disponible en: https://ac.els-cdn.com/S1877050915014787/1-s2.0S1877050915014787-main.pdf? tid=1bd56790-d3b411e7-9b67-00000aab0f02\&acdnat $=1511815797 \_d 51 e 4 b-$ c601e18e4ce25f62ab7eacedb0

[17] J. F. Rubiano, C. A. Peña y E. Martínez, "Avance en el desarrollo de una plataforma de control metal de un robot paralelo tipo Delta," in VII Congreso Bolivariano de Ingenieria Mecanica, 2012. http://congreso.pucp.edu.pe/ vii-cobim/H.1.11.pdf

[18] B. Nakisa, M. N. Rastgoo, D. Tjondronegoro y V. Chandran, "Evolutionary Computation Algorithms for Feature Selection of EEG-based Emotion Recognition using Mobile Sensors," Expert Syst. Appl., 2017. DOI: 10.1016/j.eswa.2017.09.

[19] T. H. E. Science, "Open Your Mind to Next Generation Brainwe EMOTIV Insight Introduction Vid,” 2017. [En línea]. Disponible en: https://www.emotiv.com/insight/ HARDWARE

[20] C. Peña, S. Caicedo, L. U. Z. Moreno, M. Maestre y A. Pardo, "Use of a Low Cost Neurosignals Capture System to Show the Importance of Developing Didactic Activities Within a Class to Increase the Level of Student Engagement (Case Study)," WSEAS Trans. Comput., vol. 16, pp. 172-178, 2017. http://www.wseas.org/multimedia/journals/computers/2017/a385905-070.php

[21] C. A. Peña Cortés, O. E. Gualdrón y G. G. Moreno Contreras, "Warning and Rehabilitation System Using Brain Computer Interface (BCI) in Cases of Bruxism," Ing. y Univ., vol. 18, no. 1, pp. 177-193, 2014. DOI: 10.11144/ Javeriana.IYU18-1.sarb

[22] M. Soler, H. Rodríguez y C. Peña, "Desarrollo de un robot explorador operado mediante neuroseñales," Rev. Politécnica, vol. 10, no. 19, pp. 125-134, 2014. http://revistas.elpoli.edu.co/index.php/pol/article/view/479 